\title{
A pair of Seyferts identified from a ROSAT bright source
}

\author{
J.-H. Wu ${ }^{1}$, Q.-R. Yuan ${ }^{2}$, X.-T. He ${ }^{3}$, W. Voges ${ }^{4}$, R.-D. Nan ${ }^{1}$, R. F. Green ${ }^{5}$, and X.-Z. Zhang ${ }^{1}$ \\ 1 National Astronomical Observatories, Chinese Academy of Sciences, A20 Datun Road, Beijing 100012, China \\ 2 Department of Physics, Nanjing Normal University, Nanjing 210097, China \\ 3 Department of Astronomy, Beijing Normal University, Beijing 100875, China \\ 4 Max-Planck-Institute für Extraterrestrische Physik, 85740 Garching, Germany \\ ${ }^{5}$ Kitt Peak National Observatory, NOAO, Tucson, AZ85726-6732, USA
}

Received 25 May 2001 / Accepted 26 September 2001

\begin{abstract}
We report the discovery of a pair of Seyfert galaxies identified in the ROSAT bright source, 1RXS J000306.3-041932. The two Seyferts have the same redshift of 0.106. The angular distance between them is $\sim 23^{\prime \prime}$, corresponding to a projected separation of $\sim 73 \mathrm{kpc}$, and suggesting an interacting system. We argue, from its weak IR emission and relatively large nuclear separation, that the system is an example of the interaction between AGNs, rather than interaction-triggered AGN activity.
\end{abstract}

Key words. galaxies: interactions - galaxies: Seyfert - X-rays: galaxies

\section{Introduction}

A large number of galaxies show very peculiar morphologies such as shells, bridges, tidal tails, or multiple nuclei (Arp 1966; Schweizer 1990). They are believed to be interacting or merging systems. As a kind of common phenomenon in the Universe, galaxy interactions and mergers are now recognized as one of the dominant processes governing galaxy formation and evolution (Barnes \& Hernquist 1992).

Galaxy interactions and mergers are usually associated with strong infrared emission. Starburst activity is frequently identified in this kind of system. Models of starbursts triggered by galaxy interactions and mergers have been proposed (Joseph 1990; Sanders \& Mirabel 1996). Several authors have also suggested that galaxy interactions and mergers might be responsible for AGN activity (Toomre \& Toomre 1972). Indeed, many AGNs have close companions or show very disturbed morphologies (e.g. Keel 1996; Bahcall et al. 1997).

However, few AGNs have been found in strongly interacting or merging systems, and binary AGNs are even more rare. To date only a dozen or so binary Seyferts have been found in an optical survey (Schneider et al. 1994). During a program of identifying the ROSAT bright sources that have no counterparts in any other existing catalog we found 1RXS J000306.3-041932 to be associated with a pair of Seyferts at redshift 0.106. The proper distance

Send offprint requests to: J. -H. Wu, e-mail: jhwu@bao.ac.cn
Table 1. X-ray data on 1RXS J000306.3-041932.

\begin{tabular}{lll}
\hline \hline Parameter & Value & \\
\hline position (J2000) & 000306.31 & -41932.16 \\
count rate $($ counts s & \\
hardness ratio 1 & 0.0772 & \\
hardness ratio 2 & -0.43 & \\
flux $\left(\mathrm{erg} \mathrm{s}^{-1} \mathrm{~cm}^{-2}\right)$ & +0.21 \\
luminosity $\left(\mathrm{erg} \mathrm{s}^{-1}\right)$ & $1.798 \times 10^{-12}$ \\
source extension & $37^{\prime \prime}$ \\
\hline
\end{tabular}

between them suggests an interacting system. This paper presents our observations and analyses of this system.

\section{Spectroscopic observations and results}

1RXS J000306.3-041932 is a "blank field" source in the ROSAT All-Sky Survey (RASS) Bright Source Catalog, since no counterpart is found in any other catalog within a search radius of $300^{\prime \prime}$ around it. Its X-ray data are listed in Table 1. The flux and luminosity were calculated by assuming a power-law spectrum $F_{\text {photon }} \propto E^{-\Gamma}$. The X-ray photon index was estimated from the two hardness ratios as $\Gamma=3.48$ if it is assumed to be due only to Galactic absorption. The column density of hydrogen absorption was estimated as $3.45 \times 10^{20} \mathrm{~cm}^{-2}$ as measured by $21 \mathrm{~cm}$ radio observations.

The optical spectroscopic observation was carried out at the Xinglong Station, Beijing Astronomical 

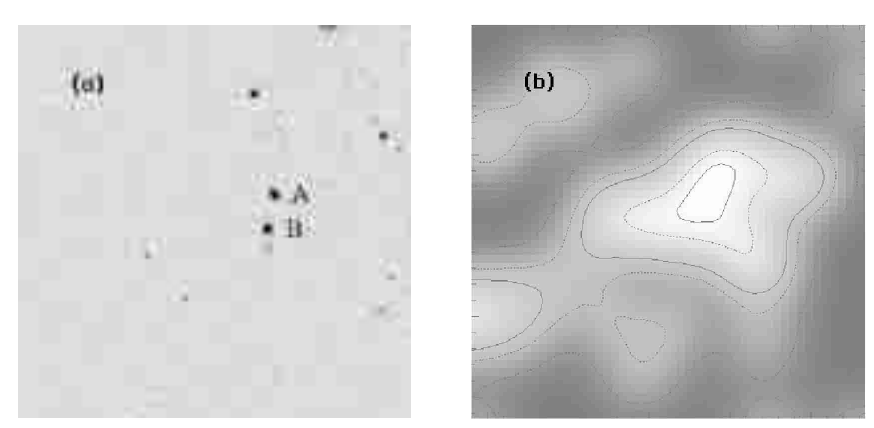

Fig. 1. Panel a): finding chart of 1RXS J000306.3-041932; panel $\mathbf{b}$ ): the broad-band X-ray contour image. Both images are $4^{\prime} .2 \times 4^{\prime} .2$ and centered on the cataloged X-ray position. East is to the left and south to bottom.

Observatory on December 16, 1998. We used the $2.16 \mathrm{~m}$ telescope and OMR spectrograph. The finding chart was obtained from the digitized Palomar Optical Sky Survey (DSS) and is shown in Fig. 1a. Two objects lie about $34^{\prime \prime}$ west of the X-ray position. Since the RASS has a positional uncertainty of $\sim 30^{\prime \prime}$ (Voges et al. 1992) and no other objects can be found closer to the X-ray position than these two objects, the two were assumed to be the optical counterparts of the X-ray source. USNO-A2.0 (Monet et al. 1996) gives their magnitudes as $B=17.1$ for Object $A$ (the northern object) and $B=16.9^{1}$ for Object $\mathrm{B}$ (the southern one). Because the two magnitudes are similar, they were put in one slit and exposed simultaneously. We used the grating with $195 \AA / \mathrm{mm}$ and Tektronix $1024 \times 1024$ CCD detector. An exposure of 2400 s gave a spectral coverage of $4000 \sim 8500 \AA$ and a spectral resolution of $\sim 15 \AA$. Data reduction was done with IRAF and the final spectra are shown in Fig. 2.

The $\mathrm{H} \alpha, \mathrm{H} \beta$ and [S II] lines can be seen in Object A's spectrum. For Object $\mathrm{B}$, the $\mathrm{H} \alpha, \mathrm{H} \beta$ and [O III] emission lines are clear. These lines give their redshifts both as $z=$ 0.106 , corresponding to a luminosity distance of $652 \mathrm{Mpc}$. When the USNO-A2.0 magnitudes are adopted, the absolute $B$ magnitudes are calculated as $M_{B}=-21.92$ for Object $\mathrm{A}$ and $M_{B}=-22.12$ for Object B. Neither is bright enough to be a quasar. The optical data of this system are summarized in Table 2. The optical positions are those in USNO-A2.0.

Line measurements were made for the spectra by using the task SPLOT within the IRAF environment. We applied Gaussian fitting to the emission lines. The instrumental width was determined from the $F W H M$ s of night-sky spectra taken with the scan and subtracted from the measured widths of the emission lines. Although the measurements are not very accurate, the results for Object B suggest that it should be a narrow-line Seyfert 1 galaxy (NLS1): it has $\mathrm{H} \alpha F W H M \sim 1290 \mathrm{~km} \mathrm{~s}^{-1}$ and $\mathrm{H} \beta$ $F W H M \sim 1140 \mathrm{~km} \mathrm{~s}^{-1}$. The line ratio is $[\mathrm{O} \mathrm{III}] / \mathrm{H} \beta \approx 0.9$. All are consistent with the characteristics of NLS1s.

${ }^{1}$ USNO magnitudes are measured from the scanning results of DSS plates and hence are not very accurate.
Table 2. Optical data on 1RXS J000306.3-041932.

\begin{tabular}{ccc}
\hline \hline Parameter & Object A & Object B \\
\hline RA $($ J2000) & 000303.67 & 000304.04 \\
DEC (J2000) & -41915.8 & -41938.1 \\
$B$ & 17.1 & 16.9 \\
$M_{B}$ & -21.92 & -22.12 \\
redshift & 0.106 & 0.106 \\
\hline
\end{tabular}

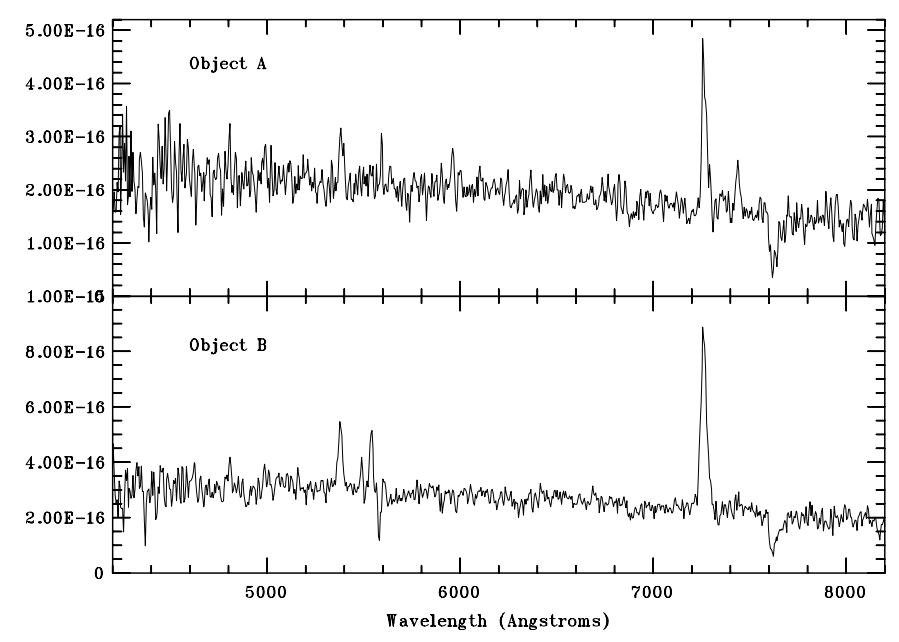

Fig. 2. Spectra of 1RXS J000306.3-041932. The flux $\left(F_{\lambda}\right)$ is in $\operatorname{erg~cm} \mathrm{cm}^{-2} \mathrm{~s}^{-1} \AA^{-1}$.

Object A, on the other hand, has a spectrum with a relatively lower signal-to-noise ratio, especially at the short wavelengths around $\mathrm{H} \beta$. Its [O III] doublets are even lost in the noise. However, it is also likely to be a NLS1 according to the line width measurements. It has $\mathrm{H} \alpha$ $F W H M \sim 960 \mathrm{~km} \mathrm{~s}^{-1}$ and $\mathrm{H} \beta F W H M \sim 1470 \mathrm{~km} \mathrm{~s}^{-1}$ (The [N II] 6583 line is barely resolved on the right wing of $\mathrm{H} \alpha$, as illustrated in Fig. 3, and was deblended from $\mathrm{H} \alpha$ when measuring the line width of $\mathrm{H} \alpha$. The [N II] 6548 line was not deblended during the measurement. However, it will contribute very little to the width and strength of the blended line $\mathrm{H} \alpha+[\mathrm{N} \mathrm{II}]$ since it is usually much weaker than the [N II] 6583 line.) These broad lines rule out the possibility that Object $\mathrm{A}$ is a starburst or $\mathrm{H}$ II galaxy. Furthermore, we can see in Fig. 1b that the X-ray peak agrees very well with the optical galaxy images in Fig. 1a in terms of both position and alignment. The north-south extension of the X-ray peak suggests that Object A will contribute significantly to the total X-ray flux. Its X-ray luminosity $\left(\sim 10^{44} \mathrm{erg} \mathrm{s}^{-1}\right)$ will be almost two orders of magnitude brighter than the brightest known X-ray luminosity for a starburst or H II galaxy, but is typical for Seyfert 1s. Thus, Object A is also most likely to be a NLS1, or something between Serfert 1.5 and Seyfert 2.

We estimated from the finding chart that the angular separation between the two objects is $\sim 23^{\prime \prime}$. This relatively large angular separation and the differences in spectra rule out the probability of a gravitationally lensed system. Nevertheless, the projected nuclear separation, 


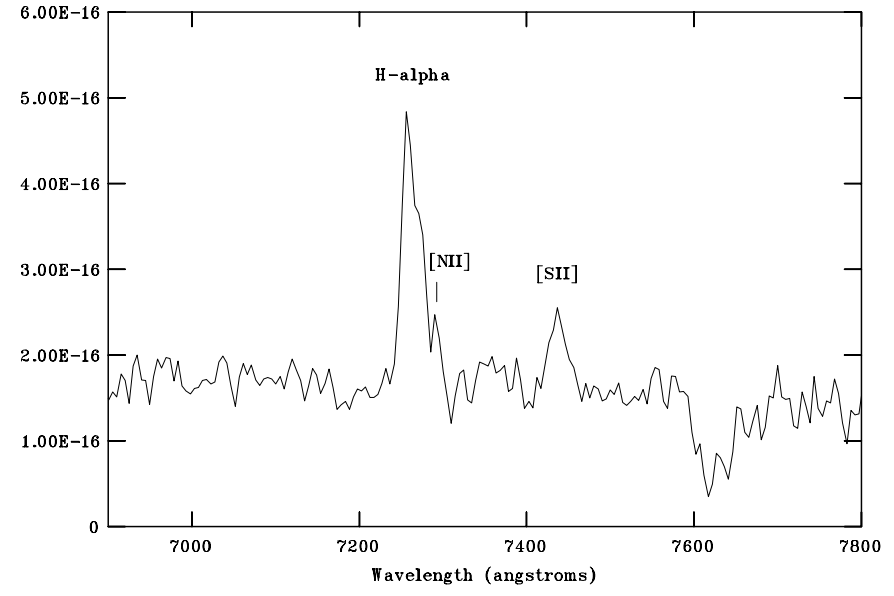

Fig. 3. Spectrum of Object A at long wavelengths. The flux $\left(F_{\lambda}\right)$ is in $\operatorname{erg~cm}^{-2} \mathrm{~s}^{-1} \AA^{-1}$.

$\sim 73 \mathrm{kpc}^{2}$, suggests an interacting system. If both objects are indeed NLS1s, this system will be the first known interacting system of two NLS1s.

To investigate the continuum energy distribution of these galaxies we have searched the NVSS (Condon et al. 1998) catalog, the IRAS Point Source Catalog and Faint Source Catalog. No counterparts were found. The radio and IR emission from the system appear weak. However, the newly-released 2MASS data (Skrutskie et al. 1997) include two near-IR objects at the positions given in Table 2.

\section{Discussion: Interaction-triggered AGNs or interaction between AGNs}

It has long been proposed that galaxy interactions and mergers might be responsible for triggering AGN phenomena (Toomre \& Toomre 1972). Indeed, a number of interacting or merging systems have AGNs as one of their components (e.g. Keel 1996) and some low redshift quasars show very disturbed morphologies in deep images (e.g. Bahcall et al. 1997). However, most strongly interacting systems (such as those studied by Arp) tend not to show AGN phenomena, and some AGNs are quite isolated objects with no obvious companions.

There may be some AGNs triggered by galaxy interactions or mergers, but it seems not to be the case in $1 \mathrm{RXS}$ J000306.3-041932. Most strongly interacting or merging systems show strong IR emission (Joseph 1990), indicating considerable starburst activity triggered by the tidal interaction. Numerical simulations of interactions have also shown how gas loses its angular momentum due to collision or dynamical friction and is funneled to the remnant center, fueling a starburst or AGN (Barnes 1990). The nuclear separation in 1RXS J000306.3-041932, $73 \mathrm{kpc}$, is almost an order of magnitude greater than the diameter of a typical galaxy. We would expect in this system that

\footnotetext{
${ }^{2}$ We adopt a cosmology with Hubble constant $H_{0}=$ $50 \mathrm{~km} \mathrm{~s}^{-1} \mathrm{Mpc}^{-1}$ and deceleration factor $q_{0}=0.5$ throughout this paper.
}

very little gas is experiencing collision or dynamical friction. This is indicated by its weak IR flux. A plausible interpretation would be that the two objects have just come into weak interaction. No starburst activity has yet appeared, and therefore the interaction is also unlikely to have triggered the AGN activity observed. Furthermore, observations have indicated that the luminosities of interacting systems increase rapidly at separations of less than a few kpc (Sanders \& Mirabel 1996), suggesting the significance of tidal forces at the same scale. However, the nuclear separation in system 1RXS J000306.3-041932 is an order of magnitude greater. Since tidal effects scale as the inverse cube of the distance, it is also unlikely that the AGN activity in this system is due to a tidal interaction.

If the two Seyferts were not triggered by the interaction in this system, 1RXS J000306.3-041932 is an example of interaction between existing AGNs. Active galaxies are also galaxies. If the interaction and merger of galaxies is a kind of common phenomenon in the Universe, interactions and mergers between AGNs or between AGNs and normal galaxies can also be expected. Those binary systems with AGNs as one or both of their components, with relatively large nuclear separations, such as those studied by Keel (1996) and those catalogued in Schneider et al. (1994), may well be cases of AGN-normal galaxy or AGN-AGN interactions.

Interactions and mergers have profound effects on the stellar and gas dynamics, effects which could plausibly either stimulate or quench AGN activity. Studies of interacting AGNs are therefore likely to enrich our understanding of the AGN phenomenon.

\section{Conclusions}

During a program of identifying the ROSAT Bright Sources in "blank fields", 1RXS J000306.3-041932 was found to be an interacting system of AGNs, and both objects are likely to be the so-called NLS1s. The relatively large nuclear separation and weak IR emission suggest that the two galaxies were undergoing Seyfert activity before they came into interaction. The current discovery is an example of interactions between AGNs.

Acknowledgements. We are grateful to Dr. W. Yuan who calculated the X-ray fluxes and luminosities for us. We acknowledge the referee Robert Joseph for valuable comments and suggestions. The DSS were produced at the Space Telescope Science Institute under US Government grant NAG W-2166. The images of these surveys are based on photographic data obtained using the Oschin Schmidt Telescope on Palomar Mountain and the UK Schmidt Telescope. IRAF is the Image Reduction and Analysis Facility, a general purpose software system for the reduction and analysis of astronomical data. IRAF is written and supported by the IRAF programming group at the National Optical Astronomy Observatories (NOAO) in Tucson, Arizona. Yuan is supported by the National Key Base Sciences Research Foundation under the contract G1999075402 and Chinese NSF under the contracts No. 19873007 and 19873018. This research is supported by the National Natural Science Foundation of China. 


\section{References}

Arp, H. 1966, ApJS, 14, 1

Bahcall, J. N., Kirhakos, S., Saxe, D. H., \& Schneider, D. P. 1997, ApJ, 479, 642

Barnes, J. E. 1990, N-Body Studies of Major Mergers in Dynamics and Interactions of Galaxies, ed. R. Weilen (Springer-Verlag), 186

Barnes, J. E., \& Hernquist, L. 1992, ARA\&A, 30, 705

Condon, J. J., Cotton, W. D., Greisen, E. W., et al. 1998, AJ, 115,1693

Joseph, R. D. 1990, Infrared Studies of Interacting and Merging Galaxies in Dynamics and Interactions of Galaxies, ed. R. Weilen (Springer-Verlag), 132

Keel, W. 1996, AJ, 111, 696
Monet, D., Bird, A., Canzian, B., et al. 1998 http://www.nofs.navy.mil/projects/pmm/a2.html

Sanders, D. B., \& Mirabel, I. F. 1996, ARA\&A, 34, 749

Schneider, D. P., Schmidt, M., \& Gunn, J. E. 1994, AJ, 107, 1245

Schweizer, F. 1990, Interactions in Our Time in Dynamics and Interactions of Galaxies, ed. R. Weilen (Springer-Verlag), 60

Skrutskie, M. F., Schneider, S. E., Stiening, R., et al. 1997, The Two Micron All Sky Survey (2MASS): Overview and Status in The Impact of Large Scale Near-IR Sky Surveys, ed. F. Garzon et al. (Kluwer, Netherlands), 25

Toomre, A., \& Toomre, J. 1972, ApJ, 178, 623

Voges, W. 1992, in Proc. of ISY Conf. Space Science, ESA ISY-3 (ESA Publications), 9 